\title{
LITERATURE REVIEW ON FAULT DIAGNOSIS AND LOCALIZATION METHOD OF AIRCRAFT CABLE
}

\author{
ZHAI Yu-Yao ${ }^{1, a *}$, GUO Gang ${ }^{2, b}$ \\ ${ }^{1}$ NetherlandsAviation University of Air Force,changchun 130022 china \\ ${ }^{2}$ Netherlands Aviation University of Air Force,changchun 130022 china \\ aewwzh12201@126.com, ${ }^{\mathrm{b}} 181557077 @ q q . c o m$
}

\begin{abstract}
Keywords: Lcable system; short-circuit fault; fault localization
Abstract. TCable system is an important part of the aircraft. As the plane "nervous system",cable system is the important role of carrying out electricity, assigned signal and transmission tasks, but also to provide contact information between each system control. The volume and weight of the cable is restricted because of the characteristic of the aircraft structure, it usually uses smaller diameter and thinner insulating layer wires, the cable is densely distributed and hidden deep in the various parts of the aircraft.It requests high integrity and security in the flight environment. In the process of flight,wire is often subjected to the influence of chemical pollution, radiation, heat and cold, electricity, vibration, friction, external force.The insulating layer will be wear, corrosion and aging,eventually led to the arc fault, timely detection of cable fault and implementation according to situation to ensure flight safety. This article expounds several kinds of commonly used wire fault localization method and compares their advantages and disadvantages, and application conditions, preparing for further research in the future .
\end{abstract}

\section{Introduction}

APeople invention and use of a cable in the 19th century. In the early 1900s, the cable used in the world's first airplane, the Wright brothers is the 1903 American-made aircraft, from then on the use of aircraft cable has been 100 years of history. Aircraft cable fault caused flight accidents are not uncommon, and even some have caused heavy casualties and incalculable economic losses. Maintenance and troubleshooting testing cable has become a problem of national attention. The initial judgment cable failure is observed through the artificial eye, the external characteristics of faults found by appearance or by the nature of the instrument test to determine fault, this method laborious and time-consuming. Thus, aircraft cable fault diagnosis and localization issues in the international arena has got people's attention by NASA, the Federal Aviation Administration and the US Department of Defense jointly held, aging aircraft in the previous session, fault location and maintenance, regarded aircraft cable fault as an important research topic ${ }^{[1]}$.

Currently scholars in the field of research has made a lot of achievements. The low degree of automation of domestic aircraft cable manufacturing, cable detection of some large aircraft OEMs and manufacturing are carried out manually, each aircraft requires the use of manual input, proofreading, analysis and editing of up to millions of cable data, not only work efficiency is not high, a large labor intensity, and higher error rate of data, increasing the difficulty of assembling the plane late, but also reduces the safety of the aircraft. And domestic air and cable testing basically using traditional methods: multimeter and special measuring instruments, these two methods have disadvantages: multimeter only a bunch of cables for each wire one by one test, spend a lot of time, reliability is not high ; and accuracy is not high. The general method uses a special instrument wired data transmission, but the aircraft cable tested while introducing a large number of cables, and easy implementation of complex operations. Some aviation sector is more developed, technologically advanced countries, the widespread use of cable harnesses harness analyzer open circuit, short circuit, insulation performance testing, these harness equipment with the production of modular, compact and intelligent. The United States, Germany and France and other developed countries, aviation cable testing already has many years of successful application experience, technology is more mature, 
common wire harness analyzer and other products used in wire test, test connectors and wiring system test ${ }^{[2]}$.Early prevention and detection of dedicated aviation cable faults and defects of the developed countries. US Federal Aviation Administration (FAA) to develop and funded research for lead testing technology, ongoing research support Utah State University is under the Naval Air Systems Command, and they combine the use of advanced sensor technology, artificial intelligent technology and control technology, and automatic test research on aviation cabling system to achieve before takeoff and flight can be cable fault detection and diagnosis, and now they study achieved great success in theory, the testing equipment embedded in aviation cabling system, and always pay attention to the health of the aviation cable. China for research in this area is still in its infancy, this technology should be applied to the actual is still a long way to go ${ }^{[3-4]}$.

\section{Cause aircraft cable fault exists}

The causes of aircraft cable failure. Cable failure caused the aircraft can be divided into the following five categories:

First contact with contaminated refers to aircraft cables in a very long time in adverse environmental and hydraulic oil, lubricating oil, grease, anti-icing agents, detergent, water, toilet or kitchen waste and other pollutants contact the wire insulating layer oxidized or corroded, reducing aircraft cable performance.

Second, the physical damage is an aircraft cable in contact with metal objects, friction with the surrounding objects, is unreasonably bundling or laying, or artificial stretching or trampled by the vibrational stress and other physical processes, so that the cable worn or broken, loose contact or damage, reduce aircraft cable performance.

Third, the time of aging is an aircraft used for a long cable because of its metals and physical and chemical properties of non-metallic materials, in hot, wet environment changes over time, produce material decomposition, volatile, making it the flexibility, strength, changes extensibility, compressibility, flexibility, conductivity, moisture resistance, etc., to reduce aircraft cable performance.

Fourth, the environmental impact refers to the geographical and meteorological reasons on the affected aircraft wire heat, cold, humidity, altitude ray particle radiation and other factors, so long polymeric chain was broken aircraft cable insulation, causing the insulation layer generation hairline cracks, resulting in lower performance aircraft cable.

Fifth, improper maintenance is an aircraft assembly wire during the assembly and maintenance, improper construction occurs artificially excessive tension on the wire, bent over, peeling size too big, too deep and so cutting size, resulting in lower performance aircraft cable.

Aircraft cable fault exists universality. According to the civil aviation maintenance experience of maintenance personnel, fault type of aircraft wire is divided into two hard failures and soft failures. Hard failure including the wires short-circuit fault and open fault, a common fault aircraft wire, directly affect the stability of the aircraft flight safety and electrical equipment ${ }^{[5-6]}$. Among them, the wires short-circuit fault is signaling caused incorrect aircraft fire and the main reason is the signal transfer circuit fault interrupt the main reason. Wear soft fault main guiding line fault, the main test is performed when the contents of periodic aircraft maintenance ${ }^{[7-8]}$. Wear although not cause significant damage to the wire in a short time, but over time, gradually increasing the area of wire wear, wear parts gradually serious, will lead to decreased conductivity of the wire will eventually result in the wire breaking and short circuit. In addition, intermittent problems are unique to the fault plane of the wire. When the lead just short-circuit failure or open circuit failure, along with strong vibration leaving apparent engine failure mode, causing intermittent signal trans mission. There are different detection methods depending on the type of fault, in this divided into the traditional detection technology and modern detection technologies.

Aircraft cable fault exists regula rity.Plane conductor across all regions of the aircraft, is connected between the various aircraft electrical equipment. Through regular aircraft wire failu re statistics show that aircraft wire failures that of a typical bathtub curve ${ }^{[9]}$. Early in the life cycle of the aircraft is likely due to improper factory as sembly lines tied or improper wire wear and wire fault 
occurs, the operating system may occur even lead failure in the second year of operation of the aircraft, and at the beginning of the use of aircraft wire failure rate higher. When the fault wire to adjust and repair of aircraft wire existed for some steady and relatively low during this period the possibility of lead failure. With the machine age of growth, due to the wire aging and cracks and wear, then the wire is also vulnerable to frequent failure. In addition, when the plane just after a large assembly and maintenance after the wire due to improper maintenance of the manufacturing process and maintenance personnel, resulting in a higher incidence of lead failure.

\section{Aircraft wire fault diagnosis and localization}

Offline Fault Location.Time-domain reflectometry: Time Domain Reflectometry method TDR (time domain reflectometry is a measure based on the high-speed pulse technology development on the generation, with convenient operation, test speed, will not cause harm to the wire, fault diagnosis is now widely applied and positioning method. Principle of time-domain reflectometry measurements is the use of low-voltage high-frequency pulse signal as the incident signal transmission, reflection signal at the change in the characteristic impedance of the cable, the characteristic impedance change information by comparing the reflected signal included in determining whether a faulty cable Testing and evaluation of the reflected signal is an important basis for reliable and efficiently carry out fault diagnosis and localization wire in aircraft wire fault diagnosis and location system based on the principles of time domain reflectometry, when the aircraft cable failure, wires characteristic impedance, reflection coefficient changes, thereby affecting the waveform of reflected signals. However, this method undue reliance on significant changes in the impedance of the cable barrier point, it is difficult to determine cause of high impedance faults and intermittent faults. and wire aircraft are non-uniform transmission line, this method is only used for uniform transmission line, which currently can not be used in aircraft wire fault location.

Frequency domain reflex measurement method: Frequency domain reflectometry methods can be divided into three kinds of measurement and continuous wave FM (frequency modulated continuous wave (FMCW), standing wave reflection (standing wave Reflectometry (SWR) and phase detect frequency reflection mode (phase detection FrequencyDomain Reflectometry referred PDFDR). They usually use fixed frequency standard sine signal as the input signal. The standing wave reflection method for measuring accuracy and the anti-jamming ability is poor, on the other hand, due to the standard sine signal in time domain corresponds to infinite periodic, the analysis of signal only limited in the range of frequency domain, cannot be used for the analysis of the time domain information of the signal, so from the signal processing point of waste part of useful information, thus limiting the frequency domain reflectometry method accuracy and precision [J]. PDFDR is a fault detection method based on the sweep frequency reflection technology, and can determine the fault distance of cable by measuring the phase difference of incident wave and reflected wave.. To be detected and cable launched a sinusoidal linear sweep signal (frequency step of a set of sine wave), these signals along the cable trans mission, at the fault point reflections, the incident signal and the reflected signal is also input mixer, the mixer output signal includes two frequency part: incident signal and reflected signal frequency; the incident signal and reflected signal frequency difference. When similar incident signal and reflected signal frequency, frequency of the output signal of the mixer mainly a DC voltage signal and the second harmonic, after a low-pass filter to obtain a with sine wave DC component, and then use a / D sampling, again through the fast Fourier transform (FFT), obtained from the peak point, the peak point are calculated that the location of the point of failure.

Low pressure pulse reflection method: Low voltage pulse reflection method this method is the guide line input low voltage pulse signal, pulse signal encountered the fault point will produce a reflection, according to the transmitted pulse and echo impulse of time difference and pulse wave velocity in the cable, can determine the distance of the point of failure, according to the waveform characteristics but also to determine the fault type low voltage pulse method can measure open circuit, short circuit, low resistance fault, not suitable for high impedance fault. In the actual measurement 
process, commonly used in low voltage pulse comparison method to determine the fault point, the fault core wire and core wire waveforms are compared, can better eliminate interference joints and determine the starting position of fault point and the point of failure.

On-line fault location methods.TGPS traveling wave ranging: The new communication technology is used in cable positioning can achieve on-line fault location, GPS is now widely try emerging online method. The application of this technology is mainly in the dual terminal positioning device.. Complete system including line signal acquisition part and man-machine interactive function of center server, a line when a fault occurs, the signal acquisition part to capture the fault signal, and application of GPS clock function of recording time, the function of the center server is sent these number according to the computational processing of the application of advanced computer technology, and ultimately achieve a fault location. The online positioning method of this method has been realized by Japanese scholars, which has raised the positioning to a new height of ${ }^{[10]}$.

Online positioning based on temperature, magnetic: The photomagnetic transducer on fault point of failure of surge current analysis processing to achieve positioning, advanced sensor is key to accurate positioning of the time difference. This system can be said that the on-line fault location of a major increase, he can quickly and accurately find fault, especially the accuracy of the positioning than other online methods have greatly improved. However, the system still can not get rid of the attenuation of the traveling wave and the interference of the outside, especially when the faults near the cable line are used, the error is very large.. For example, fiber optic distributed temperature sensor for cable fault ranging, the fiber is required to composite cable internal. Distributed optical fiber temperature sensor based on Raman (Raman) scattering phenomenon research, laser beam into fiber, by a spectrometer isolated Raman light scattering and Raman scattering light to and push derived cable operating temperature. After the fault, the fault distance is calculated by the time difference between the injection time of the laser pulse and the time difference of the reflected ray.. This method can do is not affected by electromagnetic effects, while the positioning accuracy and less injury; drawback is high cost of composite fiber optic cable, and the protection of a certain degree of difficulty, mostly used in important places.

Application real-time expert system: Expert system is a computer method, he through the simulation of expert thinking, try to solve a problem in a field. There are scholars real-time expert system is introduced into the cable fault location research, when the single-phase grounding fault occurred in transmission line, real-time data through the current transformer and voltage converter acquisition such as voltage and current fault data. After the intelligent communication transmission to the monitoring host. The expert system in the host computer analyzes the fault data, and the alarm fault alert the monitor personnel, and the location is realized by the preset knowledge base matching to realize the fault location.. It can be seen that the core of the expert system still contains the expert knowledge base of various types and different conditions. Along with the perfection of the fault statistics, the method has a greater prospect in the future.

\section{Summary}

Aircraft cable safety related to the aircraft, signal transmission, the reliability of the power supply system to strict requirements and prepare for prevention and detection, to ensure that the moment in a healthy state, should understand the airplane cable fault types and causes, master the corresponding detection and location method, once the cable fault occurred, can quickly using reasonable methods and instrument, detect and determine the location of the point of failure, troubleshooting and hidden dangers. More and more accurate cable fault location method is applied to the aircraft, to ensure the safety of the aircraft. Fault locating method described in this paper in the domestic and foreign aircraft cable fault location were obtained good application, using the precise positioning method of prediction and detection can be in the aircraft before the accident preventive, to prevent the accident occurred, which on the aircraft flight safety and the safety of the staff has important significance. 


\section{References}

[1]Captain J, Cox M. Reducing the Risk of Smoke and Fire in Transport Airplanes: Past History,C urrent Risk and Recommended Mitigations[R]. The Joint 9th NASA/FAA/Dod Conference on Aging Aircraft, March 2006.

[2]Subrata Mukherjee.Essential Tests for Structured Cables[J] .EC\&M,June,2009.

[3]P. Tsai, Y. Chung, C. Lo, and C. Furse.Mixed signal reflectometer hardwareimplementation forwire fault location[J], IEEE Sensors J., vo1.5, no. 6, pp. 1479-1482Dec. 2005.

[4]B.Waddoups.Analysis of Reflectometry for Detection of Chafed AircraftWiring Insulation[J]M. S.thesis, Dept. Elect. Comput. Eng., UtahState Univ., Logan, 2001.

[5]Vetter B. Smooth Surface Composite Insulation Wiring[J]. SAE International Journal of Aerospace, 2012, 5(2): 590-594.

[6]Parkey Ch, Hughes C, Locken N. Analyzing Artifacts in the Time Domain Waveform to LocateWire Faults[J].

IEEE Instrumentation and Measurement Magazine, 2012, 15(4): 16-21.

[7]Shi X D, Zhang L, Jing T, et al. Research of Aircraft Cable Fray Fault Detecting Method basedon Hilbert Huang Transformation[C]. 2010 8th World Congress on Intelligent Control and Automation. United States: Institute of Electrical and Electronics Engineers Inc., 2010:6988-6992.

[8]Jing T, Zhang L, Shi X D, et al. Research of Aircraft Cable Fault Detecting Method based on Hilbert Huang Transformation[J]. Advanced Materials Research, 2011, 214: 138-143.

[9]Tsai P J, Lo C, Chung Y C, et al. Mixed-Signal Reflectometer for Location of Faults on Aging Wiring[J]. IEEE Sensors Journal. 2005, 5(6): 1479-1482.

[10] Xiaohong Zhang. Xiongwei Jiang, Hengkun Xie. The Application of fiber optic Distributed Temperature Sensor to Fault Detection of XLPE Insulated Underground Cable [C].Proceedings of the 6th International Conference on Properties and Applications of Dielectric Materials,2000,6:21-26. 